\title{
Study Protocol - Insight 46 Cardiovascular: A Sub-study of the MRC National Survey of Health and Development
}

\author{
Sarah Ann Mason ${ }^{1,}$, Lamia Al Saikhan ${ }^{1,4}$, Siana Jones ${ }^{1}$, Gemma Bale $^{2}$, Sarah-Naomi James ${ }^{1,3}$, \\ Heidi Murray-Smith ${ }^{3}$, Alicja Rapala ${ }^{1}$, Suzanne Williams ${ }^{1}$, Brian Wong ${ }^{1}$, Marcus Richards ${ }^{1}$, Nick C. Fox ${ }^{3}$, \\ Rebecca Hardy ${ }^{1}$, Jonathan M. Schott ${ }^{1,3}$, Nish Chaturvedi ${ }^{1}$, Alun D. Hughes ${ }^{1}$ \\ ${ }^{1} M R C$ Unit for Lifelong Health and Ageing at UCL, Department of Population Science \& Experimental Medicine, UCL Institute of \\ Cardiovascular Science, University College London, London, UK \\ ${ }^{2}$ Department of Medical Physics and Biomedical Engineering, University College London, London, UK \\ ${ }^{3}$ Dementia Research Centre, Institute of Neurology, University College London, London, UK \\ ${ }^{4}$ Department of Cardiac Technology, College of Applied Medical Sciences, Imam Abdulrahman Bin Faisal University, 2835 King \\ Faisal Street, Dammam, Kingdom of Saudi Arabia
}

\section{ARTICLE INFO}

\section{Article History}

Received 23 January 2020

Accepted 06 April 2020

\section{Keywords}

Dementia

cognitive decline

vascular cognitive impairment

functional near infrared

spectroscopy (fNIRS)

\begin{abstract}
The commonest causes of dementia are Alzheimer's disease and vascular cognitive impairment. Although these conditions have been viewed as distinct entities, there is increasing evidence that neurodegenerative and vascular pathologies interact or overlap to cause cognitive decline, and that at least in some cases individuals at risk of cognitive decline exhibit abnormal cardiovascular physiology long before emergence of disease. However, the mechanisms linking haemodynamic disturbances with cognitive impairment and the various pathologies that cause dementia are poorly understood. A sub-sample of 502 participants from the Medical Research Council National Survey of Health and Development (NSHD) have participated in the first visit of a neuroscience sub-study referred to as Insight 46, where clinical, cognitive, imaging, and lifestyle data have been collected for the purpose of elucidating the pathological changes preceding dementia. This paper outlines the cardiovascular phenotyping performed in the follow-up visit of Insight 46, with the study participants now aged 74 . In addition to standard cardiovascular assessments such as blood pressure measurements, echocardiography, and electrocardiography (ECG), functional Near Infrared Spectroscopy (fNIRS) has been included to provide an assessment of cerebrovascular function. A detailed description of the fNIRS protocol along with preliminary results from pilot data is presented. The combination of lifestyle data, brain structure/ function, cognitive performance, and cardiovascular health obtained not only from Insight 46, but also from the whole NSHD provides an exciting opportunity to advance our understanding of the cardiovascular mechanisms underlying dementia and cognitive decline, and identify novel targets for intervention.
\end{abstract}

(C) 2020 Association for Research into Arterial Structure and Physiology. Publishing services by Atlantis Press International B.V. This is an open access article distributed under the CC BY-NC 4.0 license (http://creativecommons.org/licenses/by-nc/4.0/).

\section{INTRODUCTION}

The number of adults over 80 years of age is expected to increase threefold from 2015 to 2050 [1]. Dementia is one of the most important health challenges facing older adults, as an estimated $20-30 \%$ of people aged 85 and above are living with this condition $[2,3]$, and two to four times as many experience mild cognitive impairment, a strong predictor of dementia [4,5]. There is currently no cure or proven preventative treatment for cognitive decline or dementia.

The commonest causes of dementia are Alzheimer's Disease (AD) and Vascular Cognitive Impairment (VCI). In the past these

"Corresponding author.Email: s.mason@ucl.ac.uk

Peer review under responsibility of the Association for Research into Arterial Structure and Physiology

Data availability statement: Data used in this publication are available to bona fide researchers upon request to the NSHD Data Sharing Committee via a standard application procedure. Further details can be found at http://www.nshd.mrc.ac.uk/data. Doi numbers: 10.5522/NSHD/Q101; 10.5522/NSHD/Q102; and 10.5522/NSHD/Q103. conditions have been viewed as distinct entities, with AD having a neurodegenerative basis (i.e. accumulation of $\beta$-amyloid and neurofibrillary tau tangles leading to neuronal cell loss), and VCI having a vascular basis (i.e. cerebral microbleeds, leukoaraiosis, and cortical infarcts) [6]. However, there is an increasing evidence that neurodegenerative and vascular pathologies interact or overlap to cause cognitive decline. For example, cerebral hypo-perfusion is present in both vascular and non-vascular dementia [7], cortical microbleeds related to amyloid angiopathy [8], and environmental risk factors for vascular disease may be associated with $\mathrm{AD}$, and may also act synergistically with $\mathrm{AD}$ pathology to contribute to cognitive decline $[9,10]$. Several studies $[11-14]$ have also shown that cardiac dysfunction and adverse hemodynamic patterns are associated with cognitive impairment, seemingly independent of other indices of macro- and micro-vascular disease.

Neurovascular coupling lies at the interface between the cardiovascular system and neurocognitive function, directly linking neuronal activity to local perfusion. It has been shown that dysregulation 
of neurovascular coupling results in abnormal cerebral blood flow to the neurovascular unit, thereby resulting in compromised oxygen/nutrient delivery, inefficient removal of toxic metabolites, and defects in the blood-brain barrier [15]. It is hypothesized that this may accelerate net deposition of $\beta$-amyloid and neurodegeneration, potentially accounting for synergy and a bi-directional relationship between vascular and neurodegenerative pathologies $[16,17]$. Although there is growing evidence suggesting that at least some individuals at risk of cognitive decline exhibit abnormal cardiovascular physiology long before emergence of disease [18-21], mechanisms linking haemodynamic disturbances with cognitive impairment and dementia are still poorly understood.

There are many well-established methods for assessing cardiovascular health, such as measurements of blood pressure, arterial stiffness, atherosclerotic burden, exercise capacity, and cardiac function and structure. Several methods for measuring cerebrovascular function exist, and include Transcranial Doppler (TCD), Positron Emission Tomography (PET), and functional Magnetic Resonance Imaging (fMRI). However, TCD can only be used to assess blood flow in the basal arteries in the brain, and TCD, PET, and $\mathrm{FMRI}$ are not performed in a naturalistic environment, thereby limiting the types of psychometric tests that can be performed whilst scanning. Functional Near Infrared Spectroscopy (fNIRS) is an emerging technology that provides low-cost, non-invasive assessment of cerebral haemodynamics, which can be performed during cognitive and functional tests in a naturalistic environment. fNIRS is described extensively elsewhere [22], but briefly, this technique measures changes in the concentration of Oxygenated Haemoglobin (OHB) and Deoxygenated Haemoglobin (HHB) in the superficial layer of the cerebral cortex in response to cognitive stimuli. In healthy neurovascular coupling, an increase in localised blood flow in response to a stimulus exceeds the metabolic demand, leading to an increase in the concentration of $\mathrm{OHB}$ and a decrease in concentration of HHB. The resulting Haemodynamic Response Function (HRF) [23] is modulated according to the length of the stimulus, as shown in Figure 1. Details about the HRF are included in Supplementary data (S1). These changes in OHB and HHB concentration (or lack thereof) can be used to assess the pattern and strength of cerebral activation and to detect abnormalities in the neurovascular unit, perhaps arising from cognitive impairment.

Overall, there is a scarcity of research describing the mechanisms linking the cardiovascular system with cognitive impairment, partially due to challenges associated with measuring cerebrovascular function on a wide-scale. Although several small studies have shown a relationship between abnormal cerebrovascular function and individual biomarkers of Cardiovascular Disease (CVD) [24-27] a comprehensive evaluation of (1) cerebrovascular haemodynamics, in tandem with (2) comprehensive measures of cardiovascular health and (3) biomarkers of $\mathrm{AD}$ and cognitive function has not yet been performed in a population at risk of dementia.

Insight 46 [28] is a longitudinal two-time point sub-study of the Medical Research Council (MRC) National Survey of Health and Development (NSHD) [29] specifically designed to explore preclinical dementia and the causes and consequences of cerebrovascular and amyloid pathology on brain health. NSHD is a British birth cohort study that has followed 5362 individuals born during 1 week in March 1946 in England, Scotland and Wales [29]. This is a rich dataset including social factors, psychological measures, and physical and health measures (including cardiovascular function and cognition) collected over the course of the participants' lives. By pooling this life course data measured from NSHD with the imaging, neuropsychological, clinical, cardiovascular, and cerebrovascular assessments performed as part of Insight 46, we have a powerful opportunity to study the mechanisms underpinning the
15 second stimulus

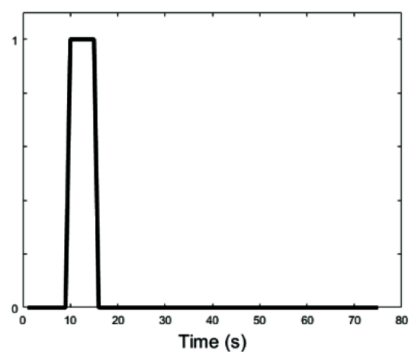

25 second stimulus

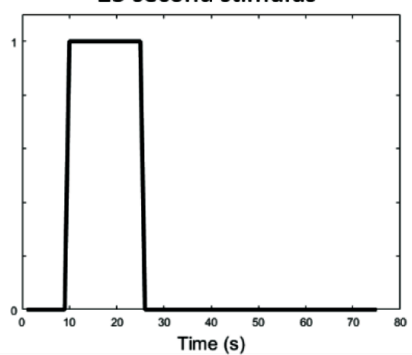

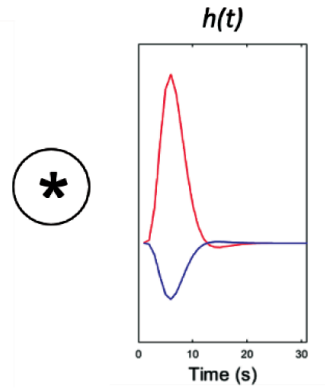

$h(t)$

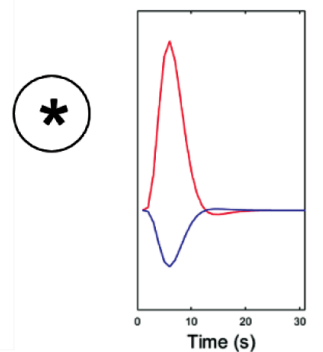

Corresponding HRF

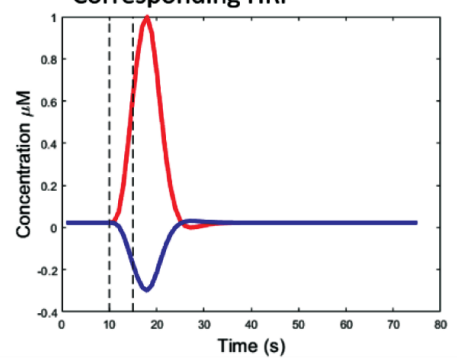

Corresponding HRF

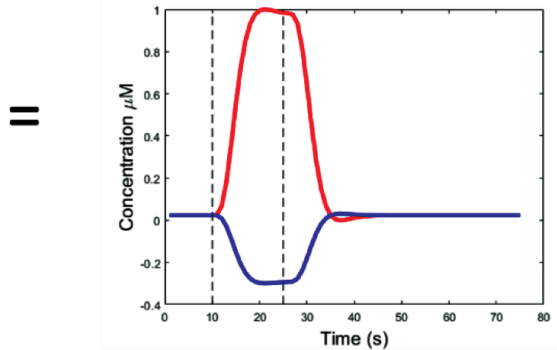

Figure 1 Demonstrates the haemodynamic response function (HRF) under different stimulus lengths. Column 1 - boxcar function, indicating onset and duration of stimulus $(y=1)$. Column 2 - canonical HRF for oxygenated haemoglobin, OHB (red) and deoxygenated haemoglobin, HHB (blue). Column 3 result of convolving boxcar stimulus function with canonical HRF in a healthy neurovascular unit. Dotted lines correspond to the onset and end of the corresponding stimulus. The $*$ symbol denotes the convolution operator. 
association between cardiovascular disease and cognitive impairment. This paper will provide an in-depth description of the cardiovascular phenotyping performed in Insight 46, with an emphasis on how fNIRS will be used to investigate cerebrovascular function.

\section{METHODS/DESIGN}

The Insight 46 sub-study of NSHD is a random subsample of 502 study participants aged 69-74 who had attended a clinic-based assessment at age 60-64 (doi: 10.5522/NSHD/Q102), had previously intimated that they were willing to attend a clinic visit in London, and for whom complete data in childhood and adulthood were available (doi: 10.5522/NSHD/Q101) [30]. Participants attended for the first round of investigation over the period 2015-2018, when they were aged 69-71 years (doi: 10.5522/NSHD/ Q103). Investigations included a cognitive testing battery and PETMRI imaging as described previously [28]. Repeat cognitive testing and PET-MRI imaging for Insight 46 commenced in 2018. This second visit was augmented by detailed cardiovascular phenotyping which included: brachial and central blood pressure measurements including and reservoir excess pressure analysis, Pulse Wave Analysis (PWA), measurements of Pulse Wave Velocity (PWV), assessment of Carotid Intimal Medial Thickness (cIMT) and carotid plaques, echocardiography, a submaximal exercise stepper test [31], a 12-lead electrocardiography (ECG) including Heart Rate Variability (HRV), and fNIRS measurements of cerebrovascular function. Each of these are described in depth in the following sections. Details of other Insight 46 investigations - including anthropometric measures, cognitive tests, PET-MRI, audiometry and the collection of wet biomarkers - can be found in the Insight 46 study protocol paper [28].

\subsection{Anticipated Sample Size and Antecedent Data}

At the time of writing, 356 participants have completed the second Insight 46 visit. Based on current rates of completion, we estimate that about 450 participants will have completed the second Insight visit by the time the study ends in the spring of 2020. Based on the first Insight 46 visit [32], we anticipate that at least $18 \%(n=81)$ of these participants will be positive for $\beta$-amyloid. By design, all participants will have complete cardiovascular data from the 60-64 NSHD clinic (as described) [29], and the majority will have complete blood pressure and anthropometric data from ages $26,43,53$, 60-64 and 69-71 in previous NSHD sweeps.

Ethical approvals for NSHD and Insight 46 were provided by Research Ethics Committees in England and Scotland as described previously [28,29,33,34]. Ethical approval for the Insight 46 Cardiovascular sub-study was given by the National Research Ethics Service Committee London (REC reference 14/LO/1173). All participants provided written informed consent to participate and for their data to be stored in accordance with the Data Protection Act.

\subsection{Brachial Blood Pressure Measurement}

Brachial blood pressure was measured in duplicate in the left arm using an HEM-907 Omron (Omron Corporation, Kyoto, Japan) with the study participant in three positions: (1) supine, (2) seated, and (3) standing. If there was a problem with the left arm (e.g. mastectomy, broken arm, etc.), the right arm was used. The following steps were repeated for each position:

- 0-3 min: participant positioned (either seated, lying down, or standing).

- 3 min: first blood pressure measurement recorded.

- 4 min: second blood pressure measurement recorded.

\subsection{Central Blood Pressure Measurements including Pulse Wave Analysis and Reservoir Excess Pressure Analysis}

Pulse wave analysis is the gold standard for non-invasively measuring central blood pressure in addition to providing relevant hemodynamic measures. We used a hand-held tonometer (SphygmoCor, AtCor Medical Pty, Sydney, Australia) to record the blood pressure waveform at the right radial artery in a semi-supine position (bed inclined at an angle or $30-45^{\circ}$ ). The waveform was calibrated to the seated systolic and diastolic brachial blood pressure measured using the MIT Elite Plus device (Omron, Kyoto, Japan). Measurements were made until a reproducible waveform was displayed for at least $12 \mathrm{~s}$. If the operator index was $<80$ the measurement was repeated until an adequate recording is achieved. The waveform overlay was also visually inspected to ensure little variability between each waveform (Figure 2). Further analysis of the blood pressure waveform will be performed using custom-written software in MATLAB to separate the pressure waveform into forward and backward components and to derive reservoir parameters [35].

\subsection{Pulse Wave Velocity}

Pulse wave velocity refers to the speed of travel of a pressure pulse along an arterial segment and is the gold standard for measuring arterial stiffness [36]. We evaluated carotid-femoral PWV using a Vicorder instrument (Skidmore Medical, Germany) to measure the transit time between a pressure sensor cuff placed over the carotid artery, and a pressure sensor cuff placed on the right thigh over the femoral artery according to recent consensus guidelines [36]. Participants were positioned with the shoulders and head raised by approximately $30^{\circ}$. To ensure an accurate measure of the path length between the two sensors, the following distance measures were performed:

1. Proximal distance (carotid): The distance from the suprasternal notch diagonally to the lower edge of the neck cuff.

2. Distal distance (femoral): The distance between the suprasternal notch directly to the top of the thigh cuff.

The waveforms from each pressure sensor were inspected to ensure each trace was noise free, and stable. If a waveform failed any of these criteria, the cuffs were readjusted, or the participant was re-positioned. We performed three repeat measurements. If the PWV varied between repeats by more than $0.5 \mathrm{~m} / \mathrm{s}$, two more measurements were acquired. If the difference between the latter three 


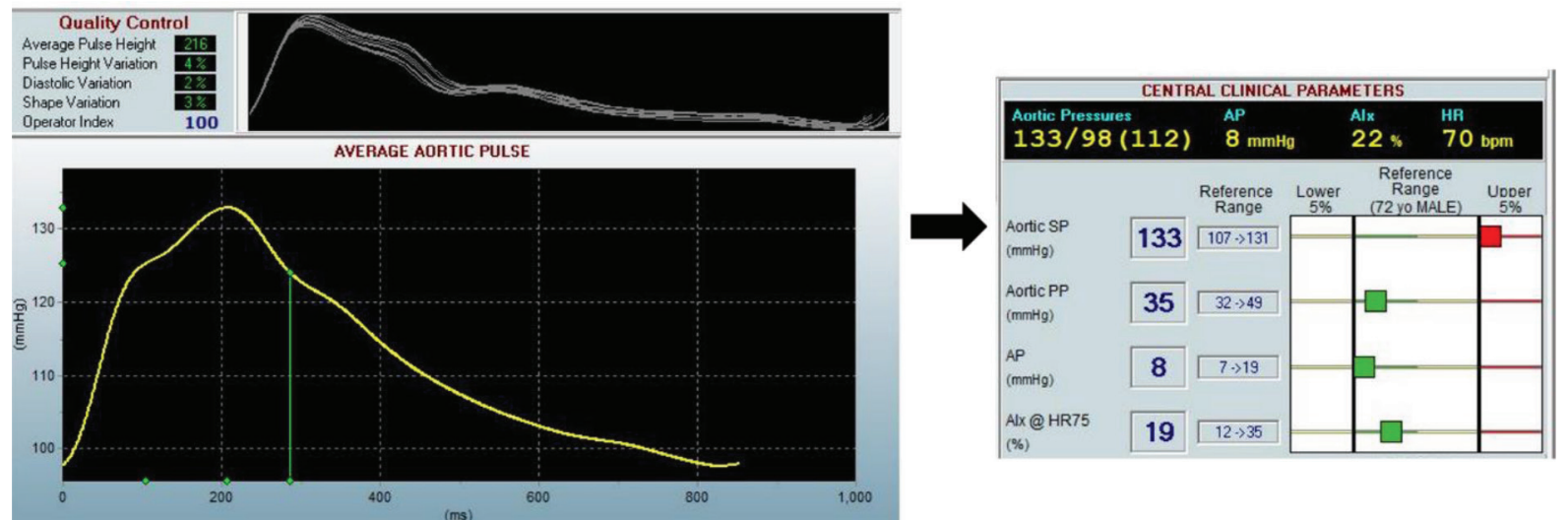

Figure 2 Example output from Sphygmocor device showing the blood pressure waveform measured at the right radial artery for participant one of Insight 46. Clinical parameters such as the aortic Systolic Pressure (SP) and aortic Pulse Pressure (PP) are calculated from the average aortic pulse waveform.

PWV measurements was still greater than $0.5 \mathrm{~m} / \mathrm{s}$, the original three measurements were accepted.

\subsection{Common Carotid Intimal Medial Thickness and Carotid Plaque}

Carotid intimal medial thickness and carotid artery plaque are used as measures of sub-clinical atherosclerosis. Vascular ultrasound of the left and right carotid arteries was performed using a EPIQ 7G scanner (Philips Healthcare, Andover MA, USA) with a linear array transducer (L12-3). A plaque was defined as a focal structure encroaching $\geq 0.5 \mathrm{~mm}$ into the arterial lumen, $\geq 50 \%$ of the surrounding IMT value, or having a thickness $>1.5 \mathrm{~mm}$ as measured from the intima-lumen interface to the media-adventitia interface [37,38]. Image acquisition settings are specified in Supplementary data (S2).

Longitudinal and transverse views of plaques were obtained and Power Doppler was used to assist in delineation of plaques. For measurement of cIMT, three cine loops of five cardiac cycles of both left and right vessels were recorded from the proximal (posterior), mid, (lateral) and distal (anterior) angulations. A still image at the $\mathrm{R}$ wave for each angle was also acquired. Analysis of far wall and near wall carotid intimal-media thickness layer will be performed over a $10-\mathrm{mm}$ straight length proximal to the bulb according to the Mannheim carotid intima-media thickness and plaque consensus recommendations [38], and measurement of plaque area, grey composition, and Gray-Weale classification will be performed using validated software (Arterial Measurement System, AMS 3.0, Chalmers University of Technology, Göteborg, Sweden) [39].

\subsection{Echocardiography}

All participants underwent transthoracic echocardiography using a Philips EPIQ 7G scanner equipped with an X5-1 transducer [see Supplementary Data (S3) for more details]. Three ECG leads were placed on the participant's chest ensuring an adequate ECG signal quality and clear QRS complex throughout the examination. Five cardiac cycles were recorded for 2D-images and 10 cardiac cycles were recorded for spectral Doppler imaging. 2D images were optimized ensuring a frame rate of at least 40 frames per second, and clear endocardial delineation before recording. Doppler acquisitions were performed with a sample volume size of 1-3 $\mathrm{mm}$ for PW Doppler and with a sweep speed ranging between 50 and $100 \mathrm{~mm} / \mathrm{s}$. For 3D-imaging, an ECG-gated full-volume 3D dataset (four sub-volumes acquired over four cardiac-cycles) of the Left Ventricle (LV) and Left Atrium (LA) was obtained. Analysis will be performed using QLab (Q-Station, version 3.9, Philips, Hamburg, Germany) for 2D and Doppler measurements and Tomtec for 3D and $4 \mathrm{D}$ measurements (TomTec Image Arena, version 2.30, TomTec Imaging Systems, Munich, Germany).

\subsection{Submaximal Exercise (Stepper) Test}

A self-paced, 6-min Stepper Test (6MST) was performed. Results from the $6 \mathrm{MST}$ have been shown to correlate closely with distance achieved in a 6-min walk test [31]. Furthermore, the 6MST permitted concurrent physiological assessments of blood pressure and heart rate using a motion-insensitive blood pressure monitor fitted with 3-lead ECG (TANGO M2, SunTech Medical Inc., Morrisville, NC, USA).

\subsection{Resting ECG and Heart Rate Variability}

A standard 12-lead ECG was performed at rest in the supine position. An additional 5-min recording of the 12-lead ECG was also collected to assess resting heart rate and HRV using a CardioPerfect ECG monitor (Welch Allyn Inc., Skaneateles Falls, NY, USA).

\subsection{Cerebrovascular Assessments using fNIRS}

To measure cerebral haemodynamics, we used a continuous wave NIRS device (Brite 24, Artinis Medical Systems, BV, Zetten, The Netherlands), which has 18 channels (10 sources and eight detectors) with wavelengths of 752 and $839 \mathrm{~nm}$ and a sampling frequency of $10 \mathrm{~Hz}$. Sixteen of the channels are referred to as 'long-separation' channels as they are separated by $3 \mathrm{~cm}$, thereby detecting light reflected from the cortical layer of the brain $(\sim 1.5 \mathrm{~cm}$ depth). These were arranged in quadrants approximately over the left and right prefrontal cortices, and the left and right motor cortices. The remaining two channels are referred to as 'short-separation' channels, as they are separated by $1 \mathrm{~cm}$, thereby detecting light reflected 
from the superficial layers of the head $(\sim 0.5 \mathrm{~cm}$ depth), including the scalp and skull. These were arranged over the left and right prefrontal cortices. fNIRS measurements were continuously recorded during three psychometric tests (see following sections for details).

\subsubsection{Spatial calibration}

Our fNIRS system was integrated with a Polhemus Patriot Digitization device (Polhemus, Colchester, VT, USA) which enables the fNIRS measurements to be spatially registered to the Montreal Neurological Institute stereotaxic coordinate system. The Polhemus works by using an electromagnetic source (located in a fixed position in the room near the head) to track the position of a sensor, which is used to label anatomical landmarks on the head (specifically the nasion, inion, left preauricular point, right preauricular point, and the central zero point) [40], and the position of each individual source and detector. The result of the digitization is illustrated in Figure 3a, where the position of each source and detector with respect to the brain was determined.

\subsubsection{Peripheral blood pressure and heart rate}

During psychometric testing (described in following section), beatto-beat peripheral blood pressure and heart rate were measured using a Finometer. To set up the Finometer, an appropriately sized cuff was selected according to the manufacturer's guidelines and fitted on the middle finger of the non-dominant hand of each participant; in some cases, the index finger was used if this provided a better fit. Electronic markers were placed to indicate the start of events within the psychometric testing protocols, thereby enabling synchronisation of hemodynamic measurements with fNIRS measurements.

An example of the experimental setup is given in Figure 3b, which shows a study participant wearing the Brite 24 fNIRS cap and the Finometer finger cuff. The orientation of the sources and detectors as measured by the Polhemus Digitization step (Figure 3a) with respect to the photograph (Figure $3 \mathrm{~b}$ ) is indicated by the magenta dashed line which corresponds to the left motor cortex in both images.

\subsection{Psychometric Testing Protocols}

We used psychometric tests that require executive and motor function as (i) these functions have been shown to be impaired in people with dementia in previous studies and (ii) the areas of the brain required to perform these functions are located near the surface, and are amenable to measurement using fNIRS.

The tests included in the fNIRS psychometric protocol are the (1) Colour Trails Test (CTT), (2) Stroop Colour Word task, and (3) finger tapping test. $\mathrm{OHB}$ and $\mathrm{HHB}$, blood pressure, and heart rate were measured continuously throughout each test using the Brite 24 and the Finometer. The testing protocols are described below.

1. Colour Trail Test (CTT): We used the CTT test developed by D'Elie and Satz where the participant was required to draw lines connecting circles in ascending order (from 1 to 25) but alternating colour (pink and yellow) [see Supplementary Data (S4)] [41-43]. Each participant was given an abbreviated practice CTT with numeric values from 1 to 8 to demonstrate their understanding of the test instructions. After the practice CTT was completed, the protocol proceeded as follows:

- 60-s baseline.

- Participant performed CTT, starting at the pink ' 1 ' and ending at the pink '25'. If they made a mistake, the operator re-directed them to the last correct value and asked them to start from there.

- 60-s recovery.

2. Stroop Colour Word task: In this test, the participant was required to read out loud either the colour of the ink or the word as it was spelled depending on the administrator's instruction [see Supplementary Data (S5)]. The Stroop Colour

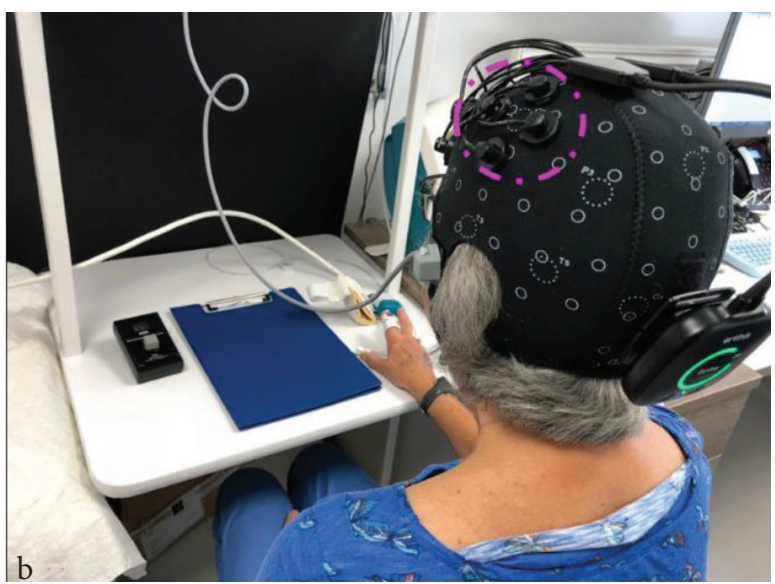

Figure 3 (a) Position of sources and detectors with respect to the head obtained using the Polhemus digitization system. The detectors are shown in blue and the sources are shown in yellow (long separation channels) and red (short separation channels). The channels are arranged in quadrants over the left and right prefrontal cortices and the left and right motor cortices. (b) Photograph showing the Brite 24 cap and the Finometer finger cuff in position on a study participant. The channels in the left motor cortex are outlined in magenta in both (a) and (b). RMC, right motor cortex; LMC, left motor cortex; RPFC, right prefrontal cortex; LPFC, left prefrontal cortex. 
Word task is a well-established psychometric test described extensively elsewhere $[44,45]$. Our protocol was comprised of three blocks, each interspersed with a 60-s baseline period where the participant was resting. The exact protocol is as follows:

- 60-s baseline.

- Block 1: Read 100 words printed on the page in black ink. The words were either 'Red', 'Green', or 'Blue'.

- 60-s recovery.

- Block 2: Read 100 'XXXX' printed on a page in coloured ink. The ink colours were red, green, and blue.

- 60-s recovery.

- Block 3 - the incongruous Stroop task: Read 100 words printed on a page in an ink colour that does not match what the word said.

- 60-s recovery.

3. Finger tapping test: An electronic tapping test device [see S6 in Supplementary Data] [46] was used to measure tapping speed by counting the number of times the button was depressed with the index finger within a 10 -s time period. Participants were instructed to lay their palm as flat as possible (with the fingers extended) on the table and device such that the index finger was the only part of the hand moving whilst tapping the device. Before setting up the fNIRS cap and the finometer, we recorded (1) the tapping speed of the participant's non-dominant hand during a single 10-s trial and (2) the tapping speed of the participant's dominant hand during a single 10-s trial. The remainder of the finger tapping protocol occurred after the fNIRS cap and Finometer had been set up, and only involved the dominant hand. Specifically, we repeated the finger tapping test five times, with short recovery periods (10-20 s) between tapping trials, and long recovery periods (60 s) before the first trial and after the fifth trial.

\subsection{1. fNIRS Signal Post-processing}

Cerebral and extracerebral tissues contribute to the overall fNIRS signal, with the latter considered a signal contaminant since it masks functional brain activity [47]. Non-evoked systemic and neuronal physiological processes (such as Mayer waves and respiration) and motion artefacts may also confound functional brain activity. To extract the component of the fNIRS signal corresponding to functional brain activity, a freely available Matlab package called HOMER2 and built-in Matlab functions were used to remove these physiological confounds and to convert the raw intensity fNIRS data to concentration using well-established fNIRS signal processing techniques. For more information on the processing steps, the reader is referred to Scholkmann et al., 2014 [22]. Our specific post-processing pipeline is described in Supplementary information (S7) $[48,49]$.

\section{INITIAL RESULTS AND PILOT DATA}

We present preliminary data from Insight 46 participants to illustrate results from the cerebrovascular assessments using fNIRS in combination with beat-to-beat blood pressure monitoring.

Figure 4 shows how Mean Arterial Pressure (MAP) changes throughout each psychometric test from a representative participant. Note how the MAP tends to increase during the task blocks (denoted by the yellow boxes superimposed over the graph) compared with the baseline periods.

Figure 5 shows the changes observed in heart rate, MAP and $\mathrm{OHB}$ measured during the Stroop task for a different study participant.
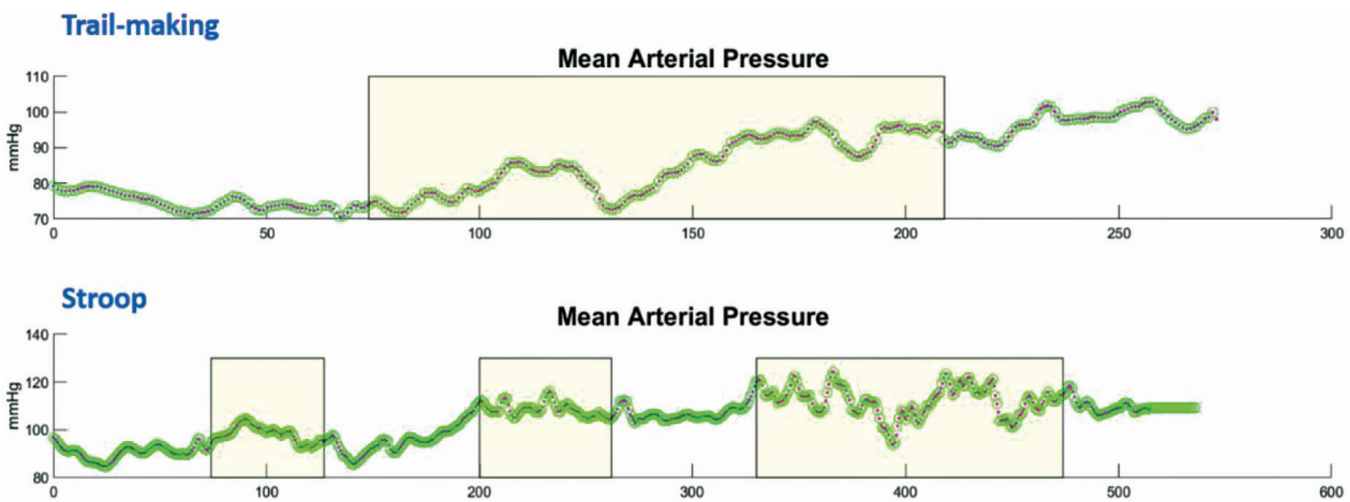

Finger-tapping

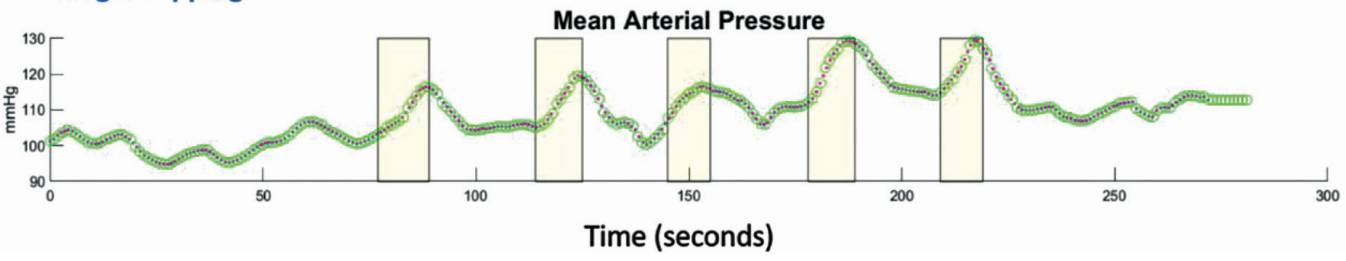

Figure 4 Change in mean arterial pressure for participant two of Insight 46 during the Colour Trail Test (CTT), Stroop, and finger tapping tasks. The yellow boxes correspond to the time periods where the participant was performing the task. 
Note that the OHB trace shown in Figure 5 is shown before the contribution of extracerebral blood flow has been regressed from the signal. All three of these cardiovascular measures tend to rise in response to the stimuli compared to the baseline periods. This figure also demonstrates the feasibility of synchronising the Finometer and the Brite 24 fNIRS cap.

Preliminary finger-tapping fNIRS results from an Insight 46 study participant considered to be in good cardiovascular health are shown in Figure 6. The block averaged fNIRS results for each channel are organised in rows according to their location over the brain. Note the strong increase in OHB in Left Motor Cortex (LMC), and the weaker but clear increase in OHB on the Right Motor Cortex (RMC) and Left Prefrontal Cortex (LPFC).

\section{DISCUSSION}

Preliminary results from this pilot data indicate that the Finometer and fNIRS are sensitive to the physiological changes induced by the psychometric tests performed in the second visit of Insight 46. The increase in MAP and heart rate suggest activation of the sympathetic nervous system during the cognitive tasks, which is in line with previously published observations [22,50].

As fNIRS is the most exploratory technique performed in Insight 46 , it is important to establish the integrity with which it can assess cerebrovascular function. The well-established activation patterns of finger tapping make this psychometric test an ideal candidate for validating the fNIRS-derived measures of neurovascular
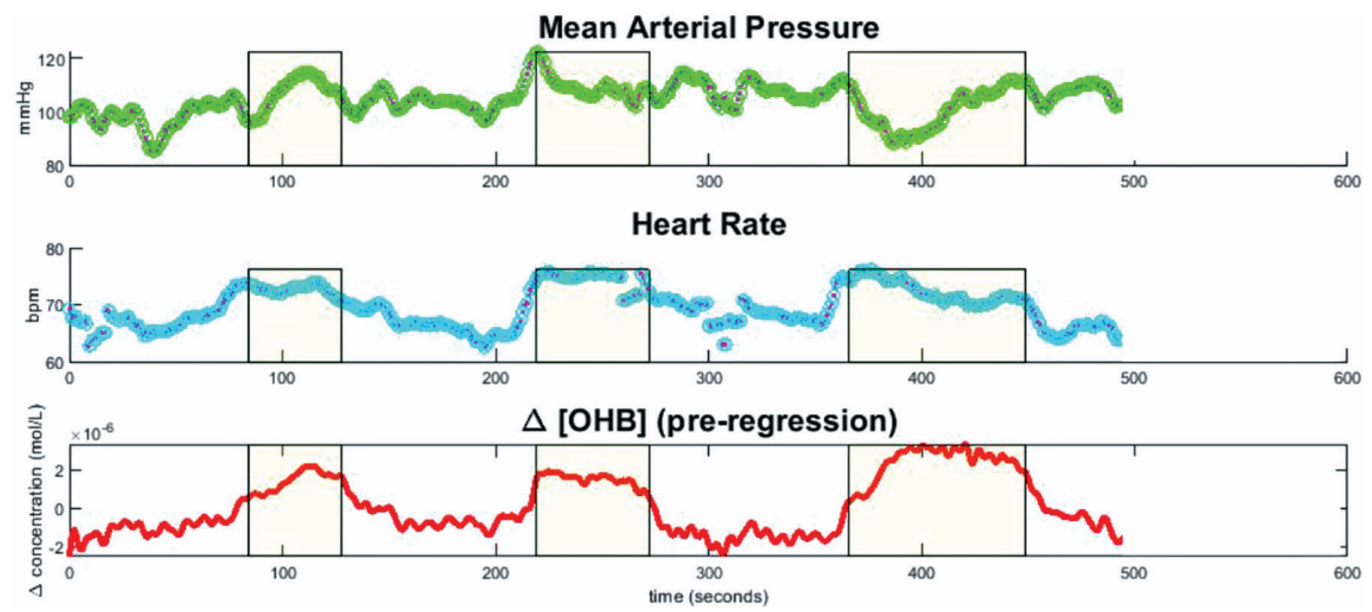

Figure 5 The change in mean arterial pressure, heart rate, and the concentration of Oxygenated Haemoglobin (OHB) measured during the Stroop task for participant three of Insight 46. Blocks 1-3 of the Stroop task (the cognitive stimuli) are denoted by the yellow rectangles superimposed over each individual graph. Note how all three cardiovascular measures increase in response to the stimuli compared with the baseline readings.

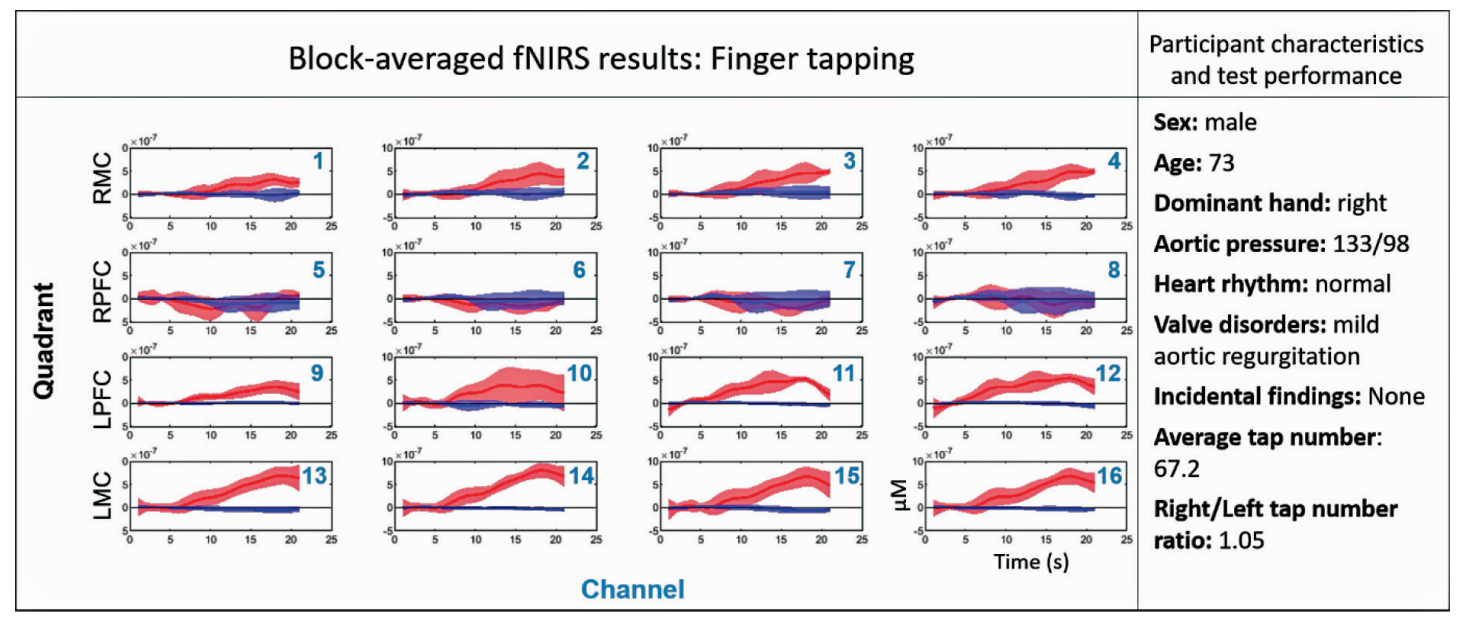

Figure 6 Results from the finger tapping test, participant characteristics, and test performance shown for participant one of Insight 46 . The cardiovascular characteristics reported were measured during the standard cardiovascular assessments using echocardiography, ECG, and PWV. The bottom right subplot indicates the axes labels for all other subplots. The red and blue solid lines correspond to the mean Oxygenated Haemoglobin (OHB) and Deoxygenated Haemoglobin (HHB) signals respectively over the five finger-tapping blocks, and the shaded areas represent the standard deviation. Finger tapping occurred over a 10-s period starting at $5 \mathrm{~s}$ and ending at $15 \mathrm{~s}$. $0-5 \mathrm{~s}$ and $15-20 \mathrm{~s}$ are baseline periods. Each of the 16 long-separation channels are displayed for each participant, and are arranged in rows corresponding to position over the brain. Row $1=$ Right Motor Cortex (RMC), row 2 $=$ Right Prefrontal Cortex (RPFC), row 3 = Left Prefrontal Cortex (LPFC), row 4 = Left Motor Cortex (LMC). 
coupling for this study. In a participant with good cognitive function, the strongest functional brain activity (i.e. neurovascular coupling) is expected to occur in the motor cortex on the contralateral side of the hand performing the tapping, with a lesser signal expected in the ipsilateral side [51,52]. Indeed, the fNIRS results shown in Figure 6 are reassuring, as the HRF measured in the LMC of this right-handed individual is characteristic of strong neurovascular coupling. As expected, weaker neurovascular coupling also occurred in the RMC of this participant during the finger tapping task. Future work will involve comparing expected patterns of functional brain activity on all three psychometric tests with fNIRS results in the participants with the best cardiovascular health to (1) ensure use of the optimal post-processing pipeline for each test and (2) to establish a benchmark with which we can compare sub-populations of Insight 46, such as those with cardiovascular disease or amyloid plaques.

The neurovascular element is critical to understanding the mechanisms of cognitive decline, yet is largely understudied. Insight 46 attempts to bridge that gap. Although further optimisation and validation of fNIRS for our specific application is still required, the promising results from the pilot data suggest that it could be a powerful tool for investigating the link between neurovascular coupling and cognitive performance. These fNIRS measurements in conjunction with task-free functional MRI measurements of cerebral blood flow (resting-state MRI arterial spin labelling) performed on both Insight 46 visits will provide an unprecedented assessment cerebrovascular function in older adults.

Detailed assessment of the cardiovascular system and particularly the integrity of the neurovascular unit will enhance the imaging and cognitive data collected as part of Insight 46, and it should provide novel insights into the interrelationships between cardiovascular health and brain ageing. The inclusion of fNIRS in a population-based study is a relatively novel approach that has the potential to provide an evaluation of cerebrovascular function on a wide-scale in a representative sample. The combination of lifestyle data, brain structure and function, cognitive performance, and cardiovascular health obtained not only from Insight 46, but also from the whole-NSHD provides an exciting opportunity to advance our understanding of the cardiovascular mechanisms underlying the commonest forms of cognitive decline and dementia and identify novel CVD targets for intervention.

\section{CONFLICTS OF INTEREST}

Nish Chaturvedi serves on a Data Safety and Monitoring Board for a clinical trial of a glucose lowering agent, funded by AstraZeneca. No other authors have competing interests.

\section{AUTHORS' CONTRIBUTION}

SAM wrote the manuscript, helped with data collection, developed fNIRS analysis pipeline, and provided results in manuscript. LAS helped with data collection, validated echocardiography protocol, and wrote supplementary data sections describing cIMT procedure and echocardiography imaging protocol. SJ contributed in data collection and development of fNIRS, exercise, and echo protocols. GB contributed in instrumentation, data collection, and analysis associated with fNIRS. SNJ helped with INSIGHT 46 design and analysis. HMS contributed in INSIGHT 46 design, execution, and participant recruitment. AR involved in INSIGHT 46 design and execution and also helped design and write protocols for cIMT brachial blood pressure, ECG, PWA and PWV. SW contributed in INSIGHT 46 data collection, including echo, ECG, PWA, PWV, and cIMT. BW contributed in design the ANIRS protocol and helped collect fNIRS data. MR contributed to the conception of the study and developed psychometric testing protocols used. NCF contributed to the conception the study, particularly on aspects relating to cognitive decline and dementia. RH contributed to the conception the study, specifically on aspects related to MRC NSHD. JMS contributed to the conception of the study, particularly in aspects relating to dementia and brain structure/function. NC contributed to the conception of the study, particularly in aspects relating to the MRC NSHD and also involved in data collection protocols. ADH contributed to the conception of the paper and the study, particularly on aspects related to cardiovascular phenotyping and also involved in data collection protocols, data analysis, and writing of the manuscript. LAS, SJ, GB, SNJ, HMS, AR, SW, MR, NCF, RH, JMS and NC contributed in revising the manuscript.

\section{FUNDING}

Insight 46 is funded by grants from Alzheimer's Research UK (ARUK-PG2014-1946, ARUK-PG2017-1946 PIs Schott, Fox, Richards), the Medical Research Council Dementias Platform UK (CSUB19166 PIs Schott, Fox, Richards), the Wolfson Foundation (PR/ylr/18575 PIs Fox, Schott), the Medical Research Council (MC_UU_12019/1 PI Kuh and MC_UU_12019/3 PI Richards), the Wellcome Trust (Clinical Research Fellowship 200109/Z/15/Z Parker) and Brain Research Trust (UCC14191, PI Schott). The cardiovascular assessments of Insight 46 are funded by a grant from the British Heart Foundation (PG/17/90/33415 PI Hughes).

\section{ACKNOWLEDGMENTS}

We would like to thank the study members from the MRC National Survey of Health and Development (NSHD) for their lifelong commitment to the study and the Insight 46 and NSHD study teams for their work, help and guidance.

\section{SUPPLEMENTARY MATERIALS}

Supplementary data related to this article can be found at https:// doi.org/10.2991/artres.k.200417.001.

\section{REFERENCES}

[1] United Nations, Department of Economic and Social Affairs, Population Division. World Population Ageing (ST/ESA/ SER.A/390). New York: United Nations; 2015. Available from: http://www.un.org/en/development/desa/population/publications/pdf/ageing/WPA2015_Report.pdf.

[2] O’Brien JT, Erkinjuntti T, Reisberg B, Roman G, Sawada T, Pantoni L, et al. Vascular cognitive impairment. Lancet Neurol 2003;2:89-98. 
[3] World Health Organization (WHO). Dementia: a public health priority. Geneva: World Health Organization; 2012. Available from: http://apps.who.int/iris/bitstream/10665/75263/1/9789241564458_ eng.pdf [Internet] [cited Nov 5 2019].

[4] DeCarli C. Mild cognitive impairment: prevalence, prognosis, aetiology, and treatment. Lancet Neurol 2003;2:15-21.

[5] Yesavage JA, O’Hara R, Kraemer H, Noda A, Taylor JL, Ferris S, et al. Modeling the prevalence and incidence of Alzheimer's disease and mild cognitive impairment. J Psychiatr Res 2002;36:281-6.

[6] Iadecola C, Gorelick PB. Converging pathogenic mechanisms in vascular and neurodegenerative dementia. Stroke 2003;34:335-7.

[7] de la Torre JC. Cardiovascular risk factors promote brain hypoperfusion leading to cognitive decline and dementia. Cardiovasc Psychiatry Neurol 2012;2012:367516.

[8] Cordonnier C, van der Flier WM. Brain microbleeds and Alzheimer's disease: innocent observation or key player? Brain 2011;134:335-44.

[9] Breteler MM. Vascular risk factors for Alzheimer's disease: an epidemiologic perspective. Neurobiol Aging 2000;21:153-60.

[10] Dempsey RJ, Vemuganti R, Varghese T, Hermann BP. A review of carotid atherosclerosis and vascular cognitive decline: a new understanding of the keys to symptomology. Neurosurgery 2010;67:484-94.

[11] Chiesa ST, Masi S, Shipley MJ, Ellins EA, Fraser AG, Hughes AD, et al. Carotid artery wave intensity in mid-to late-life predicts cognitive decline: the Whitehall II study. Eur Heart J 2019;40:2300-9.

[12] Park CM, Williams ED, Chaturvedi N, Tillin T, Stewart RJ, Richards M, et al. Associations between left ventricular dysfunction and brain structure and function: findings from the SABRE (Southall and Brent revisited) study. J Am Heart Assoc 2017;6: pii: e004898.

[13] Jefferson AL, Beiser AS, Himali JJ, Seshadri S, O’Donnell CJ, Manning WJ, et al. Low cardiac index is associated with incident dementia and alzheimer disease: the Framingham heart study. Circulation 2015;131:1333-9.

[14] Sabayan B, van Buchem MA, Sigurdsson S, Zhang Q, Harris TB, Gudnason V, et al. Cardiac hemodynamics are linked with structural and functional features of brain aging: the age, gene/ environment susceptibility (AGES)-Reykjavik Study. J Am Heart Assoc 2015;4:e001294.

[15] Kisler K, Nelson AR, Montagne A, Zlokovic BV. Cerebral blood flow regulation and neurovascular dysfunction in Alzheimer's disease. Nat Rev Neurosci 2017;18:419-34.

[16] Beishon L, Haunton VJ, Panerai RB, Robinson TG. Cerebral hemodynamics in mild cognitive impairment: a systematic review. J Alzheimer's Dis 2017;59:369-85.

[17] Nelson AR, Sweeney MD, Sagare AP, Zlokovic BV. Neurovascular dysfunction and neurodegeneration in dementia and Alzheimer's disease. Biochim Biophys Acta 2016;1862:887-900.

[18] Kivipelto M, Ngandu T, Laatikainen T, Winblad B, Soininen H, Tuomilehto J. Risk score for the prediction of dementia risk in 20 years among middle aged people: a longitudinal, populationbased study. Lancet Neurol 2006;5:735-41.

[19] Ruitenberg A, den Heijer T, Bakker SL, van Swieten JC, Koudstaal PJ, Hofman A, et al. Cerebral hypoperfusion and clinical onset of dementia: the Rotterdam study. Ann Neurol 2005;57:789-94.

[20] Suri S, Mackay CE, Kelly ME, Germuska M, Tunbridge EM, Frisoni GB, et al. Reduced cerebrovascular reactivity in young adults carrying the APOE $\varepsilon 4$ allele. Alzheimer's Dement 2015;11:648-57.e1.
[21] Suri S, Topiwala A, Chappell MA, Okell TW, Zsoldos E, SinghManoux A, et al. Association of midlife cardiovascular risk profiles with cerebral perfusion at older ages. JAMA Netw Open 2019;2:e195776.

[22] Scholkmann F, Kleiser S, Metz AJ, Zimmermann R, Mata Pavia J, Wolf $U$, et al. A review on continuous wave functional near-infrared spectroscopy and imaging instrumentation and methodology. Neuroimage 2014;85:6-27.

[23] Aqil M, Hong KS, Jeong MY, Ge SS. Cortical brain imaging by adaptive filtering of NIRS signals. Neurosci Lett 2012;514:35-41.

[24] Braskie MN, Small GW, Bookheimer SY. Vascular health risks and fMRI activation during a memory task in older adults. Neurobiol Aging 2010;31:1532-42.

[25] Irani F, Sweet LH, Haley AP, Gunstad JJ, Jerskey BA, Mulligan RC, et al. A fMRI study of verbal working memory, cardiac output, and ejection fraction in elderly patients with cardiovascular disease. Brain Imaging Behav 2009;3:350-7.

[26] Keage HAD, Churches OF, Kohler M, Pomeroy D, Luppino R, Bartolo ML, et al. Cerebrovascular function in aging and dementia: a systematic review of transcranial Doppler studies. Dement Geriatr Cogn Dis Extra 2012;2:258-70.

[27] Haley AP, Sweet LH, Gunstad J, Forman DE, Poppas A, Paul RH, et al. Verbal working memory and atherosclerosis in patients with cardiovascular disease: an fMRI study. J Neuroimaging 2007;17:227-33.

[28] Lane CA, Parker TD, Cash DM, Macpherson K, Donnachie E, Murray-Smith H, et al. Study protocol: Insight 46 - a neuroscience sub-study of the MRC National Survey of Health and Development. BMC Neurol 2017;17:75.

[29] Kuh D, Pierce M, Adams J, Deanfield J, Ekelund U, Friberg P, et al. Cohort profile: updating the cohort profile for the MRC National Survey of Health and Development: a new clinic-based data collection for ageing research. Int J Epidemiol 2011;40:e1-e9.

[30] James SN, Lane CA, Parker TD, Lu K, Collins JD, MurraySmith $\mathrm{H}$, et al. Using a birth cohort to study brain health and preclinical dementia: recruitment and participation rates in Insight 46. BMC Res Notes 2018;11:885.

[31] Jones S, Tillin T, Williams S, Coady E, Chaturvedi N, Hughes AD. Assessment of exercise capacity and oxygen consumption using a 6 min stepper test in older adults. Front Physiol 2017;8:408.

[32] Lane CA, Barnes J, Nicholas JM, Sudre CH, Cash DM, Parker TD, et al. Associations between blood pressure across adulthood and late-life brain structure and pathology in the neuroscience substudy of the 1946 British birth cohort (Insight 46): an epidemiological study. Lancet Neurol 2019;18:942-52.

[33] Hardy R, Ghosh AK, Deanfield J, Kuh D, Hughes AD. Birthweight, childhood growth and left ventricular structure at age 60-64 years in a British birth cohort study. Int J Epidemiol 2016;45:1091-102.

[34] Kuh D, Wong A, Shah I, Moore A, Popham M, Curran P, et al. The MRC National Survey of Health and Development reaches age 70: maintaining participation at older ages in a birth cohort study. Eur J Epidemiol 2016;31:1135-47.

[35] Davies JE, Baksi J, Francis DP, Hadjiloizou N, Whinnett ZI, Manisty $\mathrm{CH}$, et al. The arterial reservoir pressure increases with aging and is the major determinant of the aortic augmentation index. Am J Physiol Heart Circ Physiol 2010;298:H580-H6.

[36] Van Bortel LM, Laurent S, Boutouyrie P, Chowienczyk P, Cruickshank JK, De Backer T, et al. Expert consensus document on the measurement of aortic stiffness in daily practice 
using carotid-femoral pulse wave velocity. J Hypertens 2012; 30:445-8.

[37] Touboul PJ, Hennerici MG, Meairs S, Adams H, Amarenco P, Bornstein N, et al. Mannheim carotid intima-media thickness consensus (2004-2006): an update on behalf of the advisory board of the 3rd and 4th Watching the Risk Symposium 13th and 15th European Stroke Conferences, Mannheim, Germany, 2004, and Brussels, Belgium, 2006. Cerebrovasc Dis 2007;23:75-80.

[38] Touboul PJ, Hennerici MG, Meairs S, Adams H, Amarenco P, Bornstein N, et al. Mannheim carotid intima-media thickness and plaque consensus (2004-2006-2011). An update on behalf of the advisory board of the $3 \mathrm{rd}, 4$ th and 5 th watching the risk symposia, at the 13th, 15th and 20th European Stroke Conferences, Mannheim, Germany, 2004, Brussels, Belgium, 2006, and Hamburg, Germany, 2011. Cerebrovasc Dis 2012;34:290-6.

[39] Wendelhag I, Liang Q, Gustavsson T, Wikstrand J. A new automated computerized analyzing system simplifies readings and reduces the variability in ultrasound measurement of intimamedia thickness. Stroke 1997;28:2195-200.

[40] Klem GH, Lüders HO, Jasper HH, Elger C. The ten-twenty electrode system of the International Federation. The International Federation of Clinical Neurophysiology. Electorencephalogr Clin Nuerophysiol Suppl 1999;52:3-6.

[41] Salthouse TA, Toth J, Daniels K, Parks C, Pak R, Wolbrette M, et al. Effects of aging on efficiency of task switching in a variant of the trail making test. Neuropsychology 2000;14:102-11.

[42] Salthouse TA. What cognitive abilities are involved in trailmaking performance? Intelligence 2011;39:222-32.

[43] Shibuya-Tayoshi S, Sumitani S, Kikuchi K, Tanaka T, Tayoshi S, Ueno S, et al. Activation of the prefrontal cortex during the
Trail-Making Test detected with multichannel near-infrared spectroscopy. Psychiatry Clin Neurosci 2007;61:616-21.

[44] Stroop JR. Studies of interference in serial verbal reactions. J Exp Psychol 1935;18:643-62.

[45] Davidson DJ, Zacks RT, Williams CC. Stroop interference, practice, and aging. Neuropsychol Dev Cogn B Aging Neuropsychol Cogn 2003;10:85-98.

[46] Schmitt L. Finger-tapping test. In: Volkmar FR, editor. Encyclopedia of Autism Spectrum Disorders. New York: Springer; 2013.

[47] Tachtsidis I, Scholkmann F. Publisher's note: false positives and false negatives in functional near-infrared spectroscopy: issues, challenges, and the way forward. Neurophotonics 2016;3:039801.

[48] Molavi B, Dumont GA. Wavelet-based motion artifact removal for functional near-infrared spectroscopy. Physiol Meas 2012;33:259-70

[49] Scholkmann F, Spichtig S, Muehlemann T, Wolf M. How to detect and reduce movement artifacts in near-infrared imaging using moving standard deviation and spline interpolation. Physiol Meas 2010;31:649-62.

[50] Kirilina E, Jelzow A, Heine A, Niessing M, Wabnitz H, Brühl R, et al. The physiological origin of task-evoked systemic artefacts in functional near infrared spectroscopy. Neuroimage 2012;61:70-81.

[51] Kim S, Ashe J, Hendrich K, Ellermann JM, Merkle H, Uğurbil K, et al. Functional magnetic resonance imaging of motor cortex: hemispheric asymmetry and handedness. Science 1993;261:615-7.

[52] Hoshiyama M, Kakigi R, Berg P, Koyama S, Kitamura Y, Shimojo M, et al. Identification of motor and sensory brain activities during unilateral finger movement: spatiotemporal source analysis of movement associated magnetic fields. Exp Brain Res 1997;115:6-14. 